\title{
Salivary Protein NISP7 of The Brown Planthopper Functions As An Effector for Mediating Tricin Metabolism in Rice Plants
}

\section{Gu Gong}

Guangdong Academy of Agricultural Sciences

Long-Yu Yuan

Guangdong Academy of Agricultural Sciences

Yi-Feng Li

Guangdong Academy of Agricultural Sciences

Hang-Xiang Xiao

Guangdong Academy of Agricultural Sciences

Yan-Fang Li

Guangdong Academy of Agricultural Sciences

\section{Yang Zhang}

Guangdong Academy of Agricultural Sciences

\section{Wei-Jian Wu}

College of Plant Protection, South China of Agricultural University

Zhen-Fei Zhang ( $\boldsymbol{Z}$ zhangzhenfei@gdaas.cn )

Guangdong Academy of Agricultural Sciences

\section{Research Article}

Keywords: Nilaparvata lugens, rice, NISP7, tricin metabolism, effector

Posted Date: July 8th, 2021

DOI: https://doi.org/10.21203/rs.3.rs-675382/v1

License: (1) (1) This work is licensed under a Creative Commons Attribution 4.0 International License. Read Full License 


\section{Abstract}

The brown planthopper (BPH), Nilaparvata lugens, is an important pest that affects rice (Oryza sativa) production in Asia. The flavone tricin (5,7,4区-trihydroxy-3囚,5》-dimethoxy flavone) is a valuable secondary metabolite commonly found in rice plants that can defend rice plants against infestation by BPH. BPH damage can reduce the metabolic level of tricin in rice. Our preliminary transcriptome research results showed that BPH salivary protein 7, NISP7, is highly responsive to tricin stimuli. However, the function of NISP7 in mediating the interaction between the rice plant and the BPH is unknown. In this study, we cloned the NISP7 gene in N. lugens and found that its mRNA level was greater in the presence of high tricin content than low tricin content, regardless of whether the BPHs were fed a rice plant diet or an artificial diet containing $100 \mathrm{mg} / \mathrm{L}$ tricin. Knocking down NISP7 resulted in BPH individuals spending more time in the non-penetration and pathway phase, and less time feeding on the phloem of rice plants. These changes decreased BPH food intake, feeding behavior, and fitness, as well as the tricin content of the rice plants. These findings demonstrate that the salivary protein NISP7 of BPH functions as an effector for tricin metabolism in rice.

\section{Introduction}

Herbivorous insects obtain nutrients from host plants. As sessile organisms, many plants have evolved chemical defense mechanisms to resist these herbivorous insects (Howe and Jander 2008). For example, plants can initiate defense mechanisms when they detect insect oral secretions or signals from damaged plant cells. Many secondary metabolites produced by plants result from this co-evolutionary struggle between herbivores and plants (Becerra et al. 2007). Recent evidence indicates that saliva-secreted proteins can mediate host plants' defense responses (Xu et al. 2019). The saliva of herbivorous insects thus plays a key role in plant-insect interactions and in mediating the defenses of host plants (Hogenhout and Bos 2011).

The brown planthopper (BPH), or Nilaparvata lugens Stål (Hemiptera: Delphacidae), is a major rice (Oryza sativa L.) pest in Asia (Ge et al. 2018). Rice plants have evolved a variety of physical and chemical defenses against the BPH. Tricin (5,7,4囚-trihydroxy-3囚,5囚-dimethoxy flavone) is a valuable secondary metabolite that is widely distributed in the stems, leaves, and hulls of many gramineous plants, including rice (Zhang et al. 2015). Previous studies show that tricin has an ecological function against the BPH by eliciting anti-feeding or anti-oviposition behavior (Adjei-Afriyie et al. 2000a, b). In rice plants, the flavone tricin 5-0-glucoside is a probing stimulant for the white-backed planthopper (Sogatella furcifera Horvath). Ling et al. (2007) show that tricin extracted from the BPH-resistant rice variety IR36 significantly reduces mortality rates and honeydew weights of BPH nymphs, and it inhibits their oviposition and feeding behaviors. Electrical penetration graph (EPG) data show that tricin significantly inhibits in vivo and in vitro movement of the BPH stylus (Zhang et al. 2015).

The BPH is a typical phloem sap-sucking insect. The first salivary protein identified in BPHs was $\beta$ glucosidase (Sōgawa 1965). Rice plants treated with $\beta$-glucosidase react with increased salicylic acid, 
ethylene, and hydrogen peroxide levels and decreased jasmonic acid content (Wang et al. 2007). Other salivary proteins, such as NIShp, NIEG1, NIMul, and NIMLP, are shown to play roles in the BPH's salivary sheath formation and feeding (Huang et al. 2015; Ji et al. 2017; Ye et al. 2017; Shangguan et al. 2017). NIEG1 degrades cellulose in the plant cell wall to allow the stylet of a BPH to reach the phloem (Ji et al. 2017). NIMul-deficient BPHs exhibit disordered development and sometimes death (Huang et al. 2017). In plants, NIMLP induces cell death, expression of defense-related genes, and callose deposition

(Shangguan et al. 2017). However, less is known about the role of salivary effectors of BPHs in mediating the chemical defenses of Rice.

In this study, we aim to fill the knowledge gap by exploring the function of the NISP7 salivary protein in mediating the interaction between rice plants and BPHs. Specifically, we assess whether NISP7 acts as an effector for mediating tricin metabolism in rice plants. Our previous study has shown that the relationship between rice plants and BPHs is an ideal model to reveal plants' defense mechanisms at the molecular level and the effects of secondary metabolites on BPHs (Jiang et al. 2019). The results of this study can help in developing novel BPH-resistant rice cultivars.

\section{Results}

\section{Characterization of NISP7}

Using the proteomics database established for BPH by our laboratory (Gong et al. 2019), we obtained a homologous fragment (312 base pairs) based on alignments with known insect transcriptome sequences. The open reading frame of the sequence was amplified via PCR with gene-specific primers. The complete sequence ( 678 base pairs) of the gene was obtained by RACE, and it comprised a 242-bp 5' untranslated region, 98-amino acid open reading frame, and 82-base pair 3' untranslated region (Fig. 1A). The predicted protein comprised 117 amino acid residues with a theoretical molecular mass of $12.88 \mathrm{kDa}$ (Supplementary Fig. S1 and S2). We designated this sequence as NISP7 (GenBank accession no. MF278695.1).

To investigate the spatio-temporal expression pattern of NISP7 in 13 different developmental stages (1st, 2nd, 3rd, 4th and 5th instar nymphs, 1st, 3rd, 5th, 7th, 9th, 11th, 13th, 15th instar adults) and various parts of the adults (head, salivary glands, ovaries, midgut, leg, and fat body) of BPH. RT-qPCR showed that $N I S P 7$ transcripts were expressed in all nymph and adult stages (Fig. 1B). The significantly higher expression in adults suggests that NISP7 plays its most important role in the adult stage. Tissue-specific expression analysis in adults showed that the NISP7 transcript levels were slightly higher in the ovaries, followed by the fat body, midgut, and salivary gland (Fig. 1C).

\section{NISP7 expression activated by tricin in different rice varieties}

Tricin concentrations in the different rice varieties and organs were different (Fig. 2). Thetricin identification results for the TN1, Mudgo, IR26, ASD7, IR36, IR56, and RH varieties were shown in Fig. 2A. The tricin concentrations in the leaves were significantly higher than those in the stems and roots in both 
the resistant $\mathrm{RH}$ and susceptible TN1 varieties. There were no significant differences in the tricin concentrations between the stems and roots (Fig. 2B). The tricin concentration in the leaves was $28.1 \pm$ $2.6 \mathrm{ng} / \mathrm{g}$ in the RH plant, which was significantly higher than that in the leaves of the TN1 plant $(P=$ $0.002)$. The tricin concentration in the RH roots differed significantly from that in the TN1 roots $(P=$ $0.004)$.

First, the optimal concentration of tricin had been chosed from two different tricin concentrations (50 $\mathrm{mg} / \mathrm{L}$ and $100 \mathrm{mg} / \mathrm{L}$ ) (Supplementary Fig. S6). The severity scores differed significantly for the tricinresistant RH population of BPHs and tricin-sensitive TN1 population of BPHs. The mean severity scores were significantly lower for the tricin-sensitive population $(P<0.001)$ than the tricin-resistant population when feeding on the $\mathrm{RH}$ rice variety (control in this study, severity score $=9.0$ ) (Fig. 2C). The NISP7 gene mRNA expression level was higher in the tricin-resistant BPHs than in the tricin-sensitive population (Fig. $2 \mathrm{C}$ ). These results suggest that NISP7 may have a role in BPH feeding and virulence. BPH nymphs fed an artificial diet containing tricin had higher NISP7transcript levels at $12 \mathrm{~h}$ and $24 \mathrm{~h}$, compared with those fed a diet without tricin $(P<0.05, P<0.01$, respectively) (Fig. 2D). Thus, the NISP7 mRNA levels were upregulated after ingesting an artificial diet containing tricin.

\section{Fitness of BPH}

To elucidate the role of NISP7 in BPHs, we synthesized dsRNA from NISP7 and injected it into 1-day-old female adults to mediate RNAi (Supplementary Fig. S3). This treatment had strong silencing effects, reducing the NISP7transcript level significantly $(\sim 86 \%)$ in the first day after treatment, compared with the levels in the control group, in those injected with dsGFP RNA $(P<0.01)$, and in those injected with dsNISP7 ( $P=0.001$ after injection with dsGFP or dsNISP7 at 2 days, $P<0.001$ after injection with dsGFP or dsNISP7 at 3 days).

BPHs subjected to the NISP7 RNAi treatment also exhibited less feeding activity, as indicated by significantly lower excretions of honeydew, compared with the two control groups $(P<0.001$ for control and dsNISP7; $P=0.003$ for dsGFP and dsNISP7), as shown in Fig. 3A. The NISP7-RNAi BPHs also had lower weight gain values, regardless of whether they were fed RH rice plants $(P<0.001$ for control and dsNISP7, $P=0.001$ for dsGFP and dsNISP7) or an artificial diet (Fig. 3B), and smaller weight gain ratios ( $P=0.006$ for the control and dsNISP7, $P=0.003$ for dsGFP and dsNISP7), as shown in Fig. 3C. Furthermore, silencing NISP7 reduced the virulence of BPH (Fig. 3D). Compared with the two control groups, BPHs injected with dsNISP7 had significantly lower survival rates from 3 to 11 days after microinjection ( $P=0.003, P=0.003$, respectively), and most died on $\mathrm{RH}$ rice by 9 days after microinjection (Fig. 3E). Similar results were obtained when BPHs injected with dsNISP7 were fed an artificial diet $(100 \mathrm{mg} / \mathrm{L})$ (Fig. 3F). The BPHs injected with dsNISP7 fed on TN1 rice and artificial diet without tricin, the mortality rate was significantly was significantly reduced (Supplementary Fig. S4 and S5).These results indicate that silencing the NISP7 gene significantly reduces feeding and performance of BPHs on rice plants.

\section{Impairment of BPH feeding}


To determine the effect of NISP7 on BPH feeding, we used the EPG technique to profile the feeding behavior of piercing-sucking insects (Yuan et al. 2020). Five main feeding phases can be distinguished by EPG: 1) the non-penetration phase, 2) the pathway phase (including penetration, salivation, stylet movement, and extracellular activity near the phloem), 3) the intracellular phase of activity in the phloem, 4) the phloem sap ingestion phase, and 5) the xylem phase. The top of Fig. 4A shows the representative EPG traces obtained from BPHs, showing the different phases. As shown in Fig. 4B, after NISP7 knockdown in female adults, the EPG waveforms obtained over $6 \mathrm{~h}$ on TN1 rice plants showed that the duration of intracellular activity in the phloem exceeded those of the dsGFP and control groups $(P=$ $0.001)$, and the durations of the non-penetration, pathway, xylem, and phloem sap ingestion phases decreased $(P<0.001, P=0.011, P=0.001, P=0.007$, respectively). On RH rice plants, the duration of the non-penetration phase increased significantly $(P<0.001)$, whereas the durations of the intracellular activity in the phloem and phloem sap ingestion phases decreased significantly over the 6-h period $(P=$ $0.017, P<0.001$, respectively), as shown in Fig. 4C. These findings indicate that BPH individuals spent less time feeding on rice plants with high tricin levels after NISP7 knockdown.

Furthermore, BPHs fed an artificial diet high in tricin produced significantly more salivary flanges than those in the control group and those fed an artificial diet containing low tricin levels $(P<0.001)$ (Fig. 5A5C). In addition, the number of salivary flanges differed significantly between the two rice plant varieties. BPHs produced $71.43 \%$ more salivary flanges on RH plants than on TN1 plants after NISP7 knockdown (Fig. 6A and 6B). Moreover, comparing salivary flanges among the control group, the numbers of salivary flanges were sparser per unit area and deeper on TN1 plants than on RH plants (Fig. 6C and 6D).

\section{Tricin metabolism mediated by NISP7}

To determine whether the salivary protein NISP7 influences the production of tricin in rice plants, we investigated tricin levels in rice plants infested by BPH adults, where the ability to produce NISP7 was silenced or not silenced. The results showed that plants fed on by control and dsGFP groups BPH had decreased tricin levels than BPH group which injected by dsNLSP7 and plants fed on by BPH adults had decreased tricin levels compared to plants not fed on by BPHs (Fig. 7A-7C). After NISP7 knockdown, the tricin level in $\mathrm{RH}$ rice plants increased by $86.36 \%(P=0.005)$ but remained unchanged in TN1 rice plants. To further characterize the physiological properties associated with the blocked synthesis of flavonoids induced by $N I S P 7$, we examined the expression levels of genes responsible for the synthesis of flavonoids in rice plants. Our results indicated that the level of tricin induced by NISP7 shared similarities with the secondary metabolite synthetase induced by the flavonoid pathway marker genes $\mathrm{CHS}$ and $\mathrm{CHI}$. Thus, NISP7 may play important roles in defense-related signal transduction (Yang et al. 2001).

\section{Discussion}

Saliva is a complex mixture of biomolecules, and it plays crucial roles in how sap-sucking insects feed on plants (Huang et al. 2015, 2017; Ji et al. 2017; Shangguan et al. 2017; Ye et al. 2017). Our experiments demonstrated that the NISP7 secretory protein in the salivary gland of BPHs is injected into rice plants during feeding. The protein encoded by NISP7 contains three unknown domains; it is unique to BPHs and 
exhibits typical tandem amino acid duplication, which is consistent with previous analyses (Jing et al. 2017). In our previous studies, we collected watery and gelling saliva from BPHs to characterize the salivary proteome (Gong et al. 2019). BPH saliva also includes a large effector repertoire involved in its interaction with rice plants, according to previously reported evaluations of the salivary proteome (Huang et al. 2016) and watery salivary proteome (Liu et al. 2016). However, for the first time, our results showed that NISP7 is involved in the interaction between $\mathrm{BPH}$ and defensive secondary metabolites in rice plants.

Tricin is an active secondary metabolic with great potential for combating BPHs by eliciting anti-feeding or anti-oviposition behavior, according to previous studies (Adjei-Afriyie et al. 2000a, b) and confirmed by our results. In the present study, we prepared a tricin-resistant BPH population that could feed on both $\mathrm{RH}$ rice (high tricin content) and TN1 rice (low tricin content). Plant-derived compounds play key roles in insect-plant interactions, and insects can adapt to changes in the levels of these compounds (Simmonds, 2003). NISP7 may act as an herbivore effector that allows BPH to overcome the defensive effect of tricin in rice plants, as indicated by our results showing that NISP7 was more highly expressed in the tricin-resistant population than in the tricin-sensitive population. NISP7 knockdown significantly reduced transcript levels and honeydew quantity by $57.69 \%$ and $63.64 \%$ in BPHs feeding on TN1 and RH rice varieties, respectively, and weight gain decreased on both the $\mathrm{RH}$ rice diet and artificial diet containing $100 \mathrm{mg} / \mathrm{L}$ of tricin. The mortality rate reduced by $5 \%$ among BPHs fed the RH rice variety $(P<0.01)$ and by $15 \%$ among BPHs fed the artificial diet containing $100 \mathrm{mg} / \mathrm{L}$ tricin $(P<0.01)$. These results are similar to those reported in studies of two other saliva genes, NIShp and NIEG1 (Huang et al. 2015; Ji et al. 2017).

Larvae release saliva into plant cells through wound sites created when they feed on the leaves of plants (Ji et al. 2013; Huang et al. 2016). After NISP7 gene knockdown in tricin-resistant BPHs, the duration of feeding on rice plants decreased significantly. BPHs fed the high-tricin $\mathrm{RH}$ rice variety spent more time in the non-penetration and pathway phases and less time feeding on phloem, thereby decreasing their food intake, weight gain, and survival rate. However, silencing NISP7 did not affect the early ability of BPH to feed on an artificial diet containing $100 \mathrm{mg} / \mathrm{L}$ tricin. These results suggest that $N / S P 7$ facilitates phloem access via cell wall penetration by interacting with tricin.

Our EPG results showed that the duration of intracellular activity in the phloem and phloem sap ingestion phases decreased significantly after NISP7 knockdown. The durations of the non-penetration and pathway phases increased significantly among BPHs that were fed either rice plant variety or an artificial tricin-rich diet. The number of salivary flanges in the two different plant types and under various tricin levels indicated that ingestion of tricin induced significant increases in salivary flanges. The expression of NISP7 in the saliva could have affected tricin-induced feeding of BPHs. Similar to other salivary proteins, the expression of NIEG1 in the fat body might be related to the detoxification of plant defense chemicals, as reported previously for some plant cell wall-degrading enzymes in insects (Calderón-Cortés et al. 2012).

The elicitation of tricin production in rice plants as a defense reaction by NISP7 shares common features with the immune responses of well-known effectors and pathogen-associated molecular processes. It 
also may be recognized by plant pattern recognition receptors, which trigger plant defensive responses. The rice plant's defense response to BPHs involves secondary metabolites, which is a common response triggered by insect feeding (Hao et al. 2008; Hogenhout and Bos 2011; Bonaventure 2012). Our results suggest that the increased duration of the non-penetration and pathway phases induced by NISP7 silencing were dependent on the tricin content. We consider that plants might respond to NISP7 via a conserved upstream component of plant signaling pathways. The expression of NISP7 decreased the expression levels of the flavonoid pathway marker genes $\mathrm{CHS}$ and $\mathrm{CH}$. $\mathrm{CHS}$ is a chitinase gene associated with flavonoid-dependent defenses, and it is induced by elicitors (Naessens et al. 2015). CHI encodes a flavanone that is also associated primarily with flavonoid synthesis (Kiba et al. 2014). These findings suggest that the defense responses triggered by NISP7 are associated with the flavonoid signaling pathway. Inhibition of the flavonoid signaling pathway promotes the synthesis of lignin, which leads to increased thickening of plant cell walls (Wu et al. 2015). A previous study showed that BPHs feeding on the $\mathrm{RH}$ rice variety could lead to thickening of the plant cell wall (Liu et al. 2015). Increasing the thickness of the cell wall on the sieve plates might occlude the sieve tubes enough to directly inhibit continuous feeding by BPHs (Hao et al. 2008). It is thus reasonable to suggest that phloem plugging also might cause dsNISP7 to perform poorly in BPHs. Further research is required to address this question.

The results obtained in this study indicate that NISP7 may be an effector involved in the interactions between rice plants and BPHs. The expression level of NISP7 was higher in the salivary gland of the tricinresistant BPH population. After NISP7 knockdown, the amount of honeydew produced by dsRNA-treated BPHs feeding on TN1 and $\mathrm{RH}$ rice varieties decreased by $57.69 \%$ and $63.64 \%$, respectively, and the virulence scores decreased by $56.16 \%$ and $75.84 \%$, respectively. NISP7 also plays an important role in $\mathrm{BPH}$ feeding, as we found that the durations of sucking and phloem feeding decreased by $47.42 \%$ and $79.36 \%$, respectively, when BPHs fed on the $\mathrm{RH}$ rice variety. The protein encoded by NISP7 can form a complex with interacting partners when secreted into rice plants.

We found that NISP7 could influence the tricin content in rice. NISP7 may activate rice defense responses by affecting the expression of flavonoid biosynthesis genes in plants. Further studies are needed to identify the interacting partner of NISP7 in rice plant varieties and to determine the role of the complex. The novel molecular interaction identified in this study may provide new insights on the interaction between $\mathrm{BPH}$ and defensive secondary metabolites in rice. Further investigation is needed to assess the usefulness of this interaction in rice agriculture.

\section{Materials And Methods}

\section{Insects and plant materials}

Rice variety Taichung Native 1 (TN1) is a BPH-susceptible rice variety with a low concentration of tricin. Rathu Heenati $(\mathrm{RH})$ has a high tricin content (Zhang et al. 2015), all plant materials to comply with relevant institutional, national, and international guidelines and legislation. Both varieties of seeds were obtained from the experimental nursery of the Plant Protection Research Institute, Guangdong 
Agricultural Academy of Science, China. All seeds for experiments were germinated on filter paper, placed in a Petri dish, and transferred to a pot (7 cm in diameter) containing multi-purpose compost. The plants were then maintained in a growth chamber at $27 \pm 0.5^{\circ} \mathrm{C}$ with a relative humidity of $70 \pm 5 \%$ and a 12-hour light/dark cycle. Plants aged 30 days were used in the experiments.

The $N$. lugens populations used for collecting secreted saliva in this study originated from Guangdong Agricultural Academy of Science, Plant Protection Research Institute, in Guangzhou, Guangdong Province, China. The tricin-resistant population was fully reared for at least 35 generations and maintained on $\mathrm{RH}$ rice plants. The insects were reared at $27 \pm 0.5^{\circ} \mathrm{C}$ at a relative humidity of $70 \pm 5 \%$. Host plants were replaced weekly. Only 1-day-old adult BPH females were selected for use in experiments.

\section{Standards and reagents}

Tricin, high-performance liquid-chromatography grade ethyl acetate (>99.8\%), and high-performance liquid-chromatography grade methanol (> 99.9\%) were purchased from Merck; MS grade methanol (> 99.9\%) from Thermo Fisher Scientific (Waltham, MA, USA); and analytical reagent methanol from Donghong Chemical Factory (Guangzhou, China). The reagents used for molecular experiments were purchased from TransGen Biotech and Genstar (Guangzhou, China). 2,4,5-7-tetra bromo fluorescein disodium salt was purchased from Shanghai Yuanye Bio-Technology Co., Ltd. (China).

\section{Reverse transcription PCR and sequence analysis of NISP7}

Newly molted 1-day-old brachypterous female adult BPHs were anesthetized with carbon dioxide for $15 \mathrm{~s}$ and dissected in phosphate-buffered saline (pH 7.4). The head, salivary gland, midgut, ovary, leg, and fat body were carefully dissected from 100 individuals under a stereomicroscope (Leica S8AP0, Wetzlar, Germany) before placing the tissues directly in $200 \mu \mathrm{L}$ of TRIzol for total RNA extraction. After preparing 30 replicate BPHs, total RNA was isolated using a TRIzol Total RNA Isolation Kit (Takara, Dalian, China) according to the manufacturer's instructions. The total RNA concentration was quantified, and 1,000 ng of RNA was used for reverse transcription in $20 \mu \mathrm{L}$ of reaction volume using a TransScript® II One-Step gDNA Removal and cDNA Synthesis SuperMix kit (TransGen Biotech, Beijing, China) according to the manufacturer's instructions. Next, $5^{\prime}$ and $3^{\prime}$ rapid amplifications of cDNA ends (RACE) was conducted to amplify NISP7 cDNA using the 5'-full RACE kit and 3'-full RACE core set (TaKaRa, Kyoto, Japan) according to the manufacturer's instructions. Supplemental Table S1 shows the specific primers for NISP7.

PCR products were cloned into carriers using a pEASY®-Blunt Zero Cloning Kit (TransGen Biotech,Beijing, China) and sequenced by IGEBio Technology Ltd. (Guangzhou, China). The full-length cDNA sequence of NISP7 was assembled from the sequencing results and verified by PCR using the primers shown in Fig. 1. The sequence with a signal peptide was submitted to TMHMM Server version 2.0 to predict transmembrane domains (http://www.cbs.dtu.dk/services/TMHMM/). SignalP 5.0 was used to predict the presence of signal peptides (http://www.cbs.dtu.dk/services/SignalP/).

\section{RNAi}


Double-stranded RNA (dsRNA) synthesis was conducted based on the cloned NISP7 sequences. A nucleotide sequence (around 300 bp long) specific to the target gene was cloned into the pEASY® Blunt Zero vector (TransGen Biotech, Guangzhou, China) and submitted for sequencing to IGE Biotechnology Ltd. (Beijing, China). Aequorea victoria green fluorescent protein (GFP) was used as a control. PCR products were used as templates for dsRNA synthesis using a MEGAscript T7 transcription kit (Thermo Fisher, Waltham, MA, USA). Supplemental Table S1 shows the specific primers used to generate these DNA templates.

Newly molted 1-day-old brachypterous female adults were first anesthetized with carbon dioxide for $15 \mathrm{~s}$ and then injected with approximately $100 \mathrm{ng}$ of dsRNA using a Nanoject II Auto-Nanoliter Injector (Drummond Scientific, Broomall, PA, USA). After injection, the treated BPHs were reared on RH rice at $27 \pm$ $0.5^{\circ} \mathrm{C}$ at a relative humidity of $70 \pm 5 \%$ for 3 days. The injected $\mathrm{BPH}$ s were used in the experiments.

\section{Electrical penetration graph (EPG) recording}

EPG was performed using a Giga-8 DC EPG amplifier (Wageningen Agricultural University, Wageningen, The Netherlands). At $1 \mathrm{~h}$ before the EPG experiments, the 1-day-old brachypterous female adult BPHs were provided with water on filter paper, then anesthetized with $\mathrm{CO}_{2}$ for $20 \mathrm{~s}$ (Zhang et al. 2015). Newly molted 1-day-old brachypterous female adults were selected from the insect cages and subjected to one of three different treatments: control, injection with double-stranded GFP (dsGFP), or injection with dsNISP7), with 15 replicates for each treatment. BPHs were fixed using a negative pressure device.

One end of a $3-\mathrm{cm}$ length of gold wire (diameter $=12.5 \mu \mathrm{m}$ ) was connected to an amplifier through the EPG probe. The other end was connected to the dorsum of the BPH with conductive silver glue (Wageningen Agricultural University). A copper electrode (length $=10 \mathrm{~cm}$, diameter $=2 \mathrm{~mm}$ ) was inserted into the soil in the RH or TN1 planting substrate to establish the other part of the electrical circuit. After a starvation period of $30 \mathrm{~min}$, the BPH was placed on a rice plant. All EPG experiments were recorded for 6 $\mathrm{h}$ under continuous light in a climate-controlled room. The $6 \mathrm{~h}$ of continuous EPG data starting from the beginning of feeding were analyzed using EPG Stylet + software (Wageningen Agricultural University, 2012).

The artificial diet system comprised a transparent and open cylindrical container (diameter $=6 \mathrm{~cm}$, height $=5 \mathrm{~cm}$ ) with a double layer of Parafilm PM-996 (Bemis, Oshkosh, WI, USA) covering one end of the container (Zhang et al. 2015). The experimental treatments tested two concentrations: $100 \mathrm{mg} / \mathrm{L}$ tricin and no tricin. One end of a copper wire was embedded in the solution, and the other end was wrapped around the electrode. The insect electrode and experimental control conditions were used to obtain the reference EPG data for a plant.

\section{BPH bioassays for honeydew and weight gain}

Honeydew is used to measure BPH feeding activity. Honeydew was collected as described by Pathak et al. (1982). One-day-old brachypterous female adults were injected with NISP7 or GFP dsRNA or not injected (control) and placed on RH rice plants. After 3 days, the active BPHs were placed into Parafilm 
bags $(6 \mathrm{~cm} \times 5 \mathrm{~cm})$ fixed on the stem of an RH or TN1 rice plant and maintained for $24 \mathrm{~h}$ in a climatecontrolled environment at $27 \pm 0.5^{\circ} \mathrm{C}$ with a relative humidity of $70 \pm 5 \%$ and a 12 -hour light/dark photoperiod. Twenty replicates were prepared.

To assess the effects of NISP7 knockdown on feeding, BPHs subjected to three treatments (control, injected with dsGFP, or injected with dsNISP7) were allowed to feed on different rice plant varieties or on an artificial diet. A single rice plant was placed in a round plastic tube (length $=10 \mathrm{~cm}$, diameter $=2 \mathrm{~cm}$ ) with a hole to facilitate breathing. The treated BPHs were weighed and placed in the tubes. After feeding for $24 \mathrm{~h}$, the BPHs were removed and weighed again. Changes in weight were determined for BPHs fed an artificial diet and those subjected to the three treatments. Data were collected in the same manner as for the rice-fed BPHs. Twenty replicates were prepared for each treatment.

\section{Salivary flange measurements}

A single rice plant was placed in a round plastic tube (length $=10 \mathrm{~cm}$, diameter $=2 \mathrm{~cm}$ ). One-day-old female adult BPHs subjected to one of three treatments (control, injected with dsGFP, or injected with dsNISP7) were placed in plastic tubes sealed with sponges. The BPHs were maintained in a climatecontrolled environment at $27 \pm 0.5^{\circ} \mathrm{C}$ at a relative humidity of $70 \pm 5 \%$ and a 12-h light/dark photoperiod. After $24 \mathrm{~h}, \mathrm{BPHs}$ and the rice stalks were removed. The rice stalks were soaked in Eosin $\mathrm{Y}$ for $12 \mathrm{~h}$ before observing the salivary flanges on the stalks under a stereoscope. The salivary flanges from BPHs fed an artificial diet were observed directly under a microscope. Thristy replicates were prepared for each treatment.

\section{Liquid chromatography-mass spectrometry}

Liquid chromatography and mass spectrometry analyses were conducted using a DGU-20A3 (Shimadzu, Kyoto, Japan) quaternary pump equipped with an autosampler. A Supelco Discovery C18 XDB-C18 (Agilent, Santa Clara, CA, USA) column $(4.6 \times 50 \mathrm{~mm}, 1.8 \mu \mathrm{m})$ was used at ambient temperature with a sample injection volume of $10 \mu \mathrm{L}$. The elution gradient comprised a binary solvent system with $\mathrm{H}_{2} \mathrm{O}$ (solvent A) and mass spectrometry-grade methanol (solvent B) at a constant flow rate of $800 \mu \mathrm{L} / \mathrm{min}$. Mass spectrometry and tandem mass spectrometry experiments were performed using a hybrid triple quadrupole/linear ion trap API4000 Q-Trap liquid chromatography mass spectrometer (Applied Biosystems, Carlsbad, CA, USA). The method employed to analyze tricin was as described by Zhang et al. (2015).

\section{Statistical analysis}

All data in this article are expressed as the mean \pm standard deviation. The content of tricin in different parts of different rice varieties and the expression levels of NISP7 in different BPH populations (e.g., those fed on TN1 or RH rice varieties) were determined using Student's $t$-test. Duncan's multiple range test was used to analyze the expression level of BPH after feeding on Liquid diets sac-tricin for 2, 4, 8, ,12 and 24 hours; the bioassay of BPH after RNA interference; and the changes in tricin content after feeding on 
different rice varieties. All statistical tests were performed using SPSS 17.0 software (SPSS Inc., Chicago, IL, USA).

\section{Declarations}

\section{Acknowledgements}

We thank Professor Wenqing Zhang (School of Life Sciences, Sun Yat-sen University, China) and Doctor Yangshuo Dai (School of Life Sciences, Sun Yat-sen University, China) for their guidances with our experiments. This work was supported by the National Natural Science Foundation of China (Grant No. 31501633) and the Guangdong Provincial Natural Science Foundation of China (Grant No. 2015A030313573).

\section{Authors' contributions}

ZFZ, LYY, and WJW conceived and supervised the project. ZFZ and LYY designed the experiments. GG and LYY performed most of the experiments. YFL, HXX, YFL, YZ, and WJW performed some of the experiments. ZFZ, LYY, and GG analyzed data and wrote the manuscript. All authors read and approved the manuscript.

\section{Competing interests}

The authors declare no competing interests.

\section{Ethical approval}

This article does not contain any studies with human participants or animals performed by any of the authors.

\section{References}

1. Adjei-Afriyie, F. et al. Probing stimulants from the rice plant towards the smaller brown planthopper, Laodelphax striatellus (Fallen) (Homoptera: Delphacidae). Z Naturforsch C J Biosci, 55, 1038-1044 (2000a).

2. Adjei-Afriyie, F., Kim, C. S., Takemura, M., Ishikawa, M. \& Horiike, M. Isolation and identification of the probing stimulants in the rice plant for the white-back planthopper, Sogatella furcifera (Homoptera: Delphacidae). Bioscience Biotechnology Biochemi, 64, 443-446 (2000b).

3. Becerra, J. X. The impact of herbivore-plant coevolution on plant community structure. Proc Natl Acad Sci, 104, 7483-7488 (2007).

4. Bonaventure, G. Perception of insect feeding by plants. Plant Biol, 14, 872-880 (2012).

5. Calderón-Cortés, N., Quesada, M., Watanabe, H., Cano-Camacho, H. \& Oyama, K. Endogenous plant cell wall digestion: a key mechanism in insect evolution. Ann Rev Ecolog Evol Syst, 43, 45-71 (2012). 
6. Ge, Y. et al. Silencing of mir156 confers enhanced resistance to brown planthopper in rice. Planta, 248, 813-826 (2018).

7. Gong, G. et al. Salivary proteomics response of rice brown planthopper Nilaparvata lugens (Stål) to stimulation of tricin, a key rice insect-resistant compound. J Environ Entomology, 41, 50-61 (2019).

8. Hao, P. et al. Herbivore-induced callose deposition on the sieve plates of rice: an important mechanism for host resistance. Plant Physiol, 146, 1810-1820 (2008).

9. Hogenhout, S. A. \& Bos, J. Effector proteins that modulate plant-insect interactions. Curr Opin Plant Biol, 14, 422-428 (2011).

10. Howe, G. A. \& Jander, G. Plant immunity to insect herbivores. Ann Rev Plant Biol, 59, 41-66 (2008).

11. Huang, H. J. et al. A salivary sheath protein essential for the interaction of the brown planthopper with rice plants. Mol Biol, 66, 77-87 (2015).

12. Huang, H. J. et al. Screening and functional analyses of Nilaparvata lugens salivary proteome. $J$ Proteome Res, 15, 1883-1896 (2016).

13. Huang, H. J., Liu, C. W., Xu, H. J., Bao, Y. Y. \& Zhang, C. X. Mucin-like protein, a saliva component involved in brown planthopper virulence and host adaptation. J Insect Physiol, 98, 223-230 (2017).

14. Ji, R. et al. Comparative transcriptome analysis of salivary glands of two populations of rice brown planthopper, Nilaparvata lugens, that differ in virulence. PLoS One, 8, e79612 (2013).

15. Ji, R. et al. A salivary endo- $\beta-1,4$-glucanase acts as an effector that enables the brown planthopper to feed on rice. Plant Physiol, 173, 1920-1932 (2017).

16. Jing, S. L. et al. Genomics of interaction between the brown planthopper and rice. Curr Opin Insect Sci, 19, 82-87 (2017).

17. Jiang, Y. J., Zhang, C. X., Chen, R. Z. \& He, S. Y. Challenging battles of plants with phloem-feeding insects and prokaryotic pathogens. Proc Nat Acad Sci, 116, 23390-23397 (2019).

18. Kiba, A. et al. SEC14 phospholipid transfer protein is involved in lipid signaling-mediated plant immune responses in nicotiana benthamiana. PLoS One, 9, e98150 (2014).

19. Kong, C., Xu, X. \& Zhou, B. Two compounds from allelopathic rice accession and their inhibitory activity on weeds and fungal pathogens. Phytochemistry, 65, 1123-1128 (2004).

20. Ling, B., Dong, H. X., Zhang, M. X., Xu, D. \& Wang, J. S. Potential resistance of tricin in rice against brown planthopper Nilaparvata lugens (Stal). Acta Ecol Sin, 27, 1300-1307 (2007).

21. Liu, Y. et al. A gene cluster encoding lectin receptor kinases confers broad-spectrum and durable insect resistance in rice. Nature Biotechnology, 33, 301 (2015).

22. Liu, Y. et al. Marker assisted pyramiding of two brown planthopper resistance genes, Bph3 and Bph27 (t), into elite rice. Cultivars Rice, 9, 27 (2016).

23. Moheb, A., Grondin, M., Ibrahim, R. K., Roy, R. \& Sarhan, F. Winter wheat hull (husk) is a valuable source for tricin, a potential selective cytotoxic agent. Food Chemistry, 138, 931-937 (2013).

24. Naessens, E. et al. A secreted MIF cytokine enables aphid feeding and represses plant immune responses. Curr Biol, 25, 1898-1903 (2015). 
25. Pathak, P. K. \& Heinrichs, E. A. Selection of biotype populations 2 and 3 of Nilaparvata lugens by exposure to resistant rice varieties. Environ Entomol, 11, 347-371 (1982).

26. Shangguan, X. X. et al. A mucin-like protein of planthopper is required for feeding and induces immunity response in plants. Plant Physiol, 176, 552-565 (2017).

27. Simmonds, M. S. J. Flavonoid-insect interactions: recent advances in our knowledge. Phytochem, 64, 21-30 (2003).

28. Sōgawa, K. Studies on the salivary glands of rice plant leafhoppers. Japanese J Appl Entomol Zool, 9, 275-290 (1965).

29. Stochmal, A., Simonet, A. M., Macias, F. A. \& Oleszek, W. Alfalfa (Medicago sativa L.) flavonoids. 2. tricin and chrysoeriol glycosides from aerial parts. J Agric Food Chem, 49, 5310-5314 (2001).

30. Walling, L. L. Avoiding effective defenses: strategies employed by phloem-feeding insects. Plant Physiol, 146, 859-866 (2008).

31. Wang, X., Zhou, G. X. \& Xiang, C. Y. Signal transduction pathway similar to $\beta$-glucosidase activated by brown planthopper. Sci Bull, 52, 2852-2856 (2007).

32. Wang, Y. et al. Susceptibility to neonicotinoids and risk of resistance development in the brown planthopper, Nilaparvata lugens (Stål) (Homoptera: Delphacidae). Pest Manag Sci, 64, 1278-1284 (2008).

33. Wu, X., Dodgen, L. K., Conkle, J. L. \& Gan, J. Plant uptake of pharmaceutical and personal care products from recycled water and biosolids: a review. Sci Total Environ, 536, 655-666 (2015).

34. Xu, H. X. et al. A salivary effector enables whitefly to feed on host plants by eliciting salicylic acidsignaling pathway. Proceedings of the National Academy of Sciences. 116:490-495(2018).

35. Yang, J., Zhang, J., Wang, Z., Zhu, Q. \& Wang, W. Remobilization of carbon reserves in response to water deficit during grain filling of rice. Field Crops Res, 71, 47-55 (2001).

36. Ye, W. et al. A salivary EF-hand calcium-binding protein of the brown planthopper Nilaparvata lugens functions as an effector for defense responses in rice. Scientific Rep, 7, 40498 (2017).

37. Yuan, L. Y., Hao, Y. H., Chen, Q. K., Pang, R. \& Zhang, W. Q. Pancreatic triglyceride lipase is involved in the virulence of the brown planthopper to rice plants-sciencedirect. $J$ Integrative Agric, 19, 27582766 (2020).

38. Zhang, Z., Cui, B. \& Zhang, Y. Electrical penetration graphs indicate that tricin is a key secondary metabolite of rice, inhibiting phloem feeding of brown planthopper, Nilaparvata lugens. Entom Exper Applicata, 156, 14-27 (2015).

39. Zhang, Z. et al. Evaluation of tricin, a stylet probing stimulant of brown planthopper, in infested and non-infested rice plants. J Appl Entom, 141, 393-401 (2016).

\section{Figures}


A

ATTTAATTTATTCGAATACACTCTGAAAGATTCAACAATATAAGTGGCTTGTGACTCTTGT

CTTTTGAATATAATAATTGGTTGATTAATGGTATGTAAATAGAGTTAGTTTGTATGCAATAGT

GATACATTGTTTTAGTATGCAAGTGAACATTATTTTATGAGATTGTGGGTATATAAGGGGAC

ACAGTTAAGAGTTTGTCAGTTGAGGTTGACACAAGTTTACATCACTAACAACAAAC

ATGAGGGCTGCCCTGATTCTTCTCATCGTATCTGCCATCATTATTGATTCGGCCATGGCA

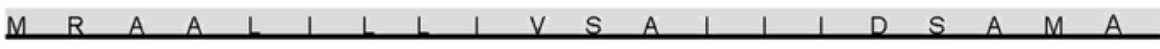

GGCCCCAAATCGAAGAAAGGCAAGAGCAAGAGGCGATCAAGGGAGAGAATCGTGTATGCA

$G \quad P \quad K \quad S \quad K \quad K \quad G \quad K \quad S \quad K \quad R \quad R \quad S \quad R \quad E \quad R \quad I \quad V \quad r \quad A$

CAGCCTCCTCCAACCCCAGTCATCATCCAAGGTGCTGCTCCATACAACTATGACAACAGA

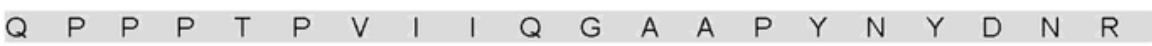

GGCTACTATGACAACAGGCCTTACCCTGGAGATGGTCGCGGATATTATGACGCCAACGGT

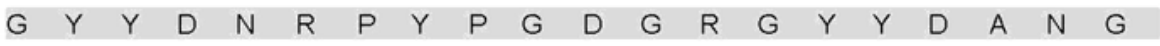

GTCTGGATCAATGGAGGCTACAATGGACCTTACCCCAATAATGGTCCAGTGGTTGTCTAC

$V W \quad I \quad N \quad G \quad G \quad Y \quad N \quad G \quad P \quad Y \quad P \quad N \quad N \quad G \quad P \quad V \quad V \quad V \quad Y$

CCCAATAATGGTCCTTATGTGCAGCCTACCTATGGACCACAGGTTGTCTACTAGATATGC

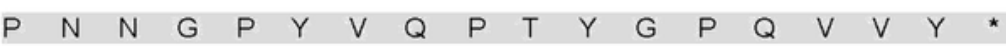

ATAATCCAGTGTTTTATGCTCCACACTACATTCTGTGTGACTTGAAGTGAGAATATAAGT

TATTGAAAAACATAAT

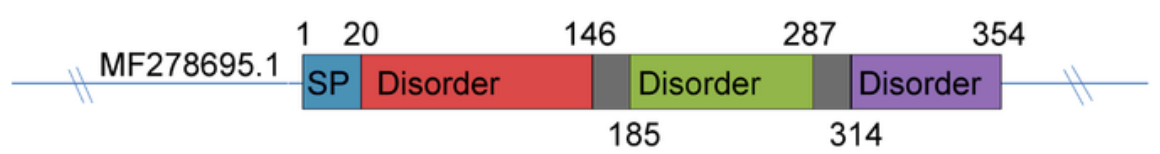

B

C
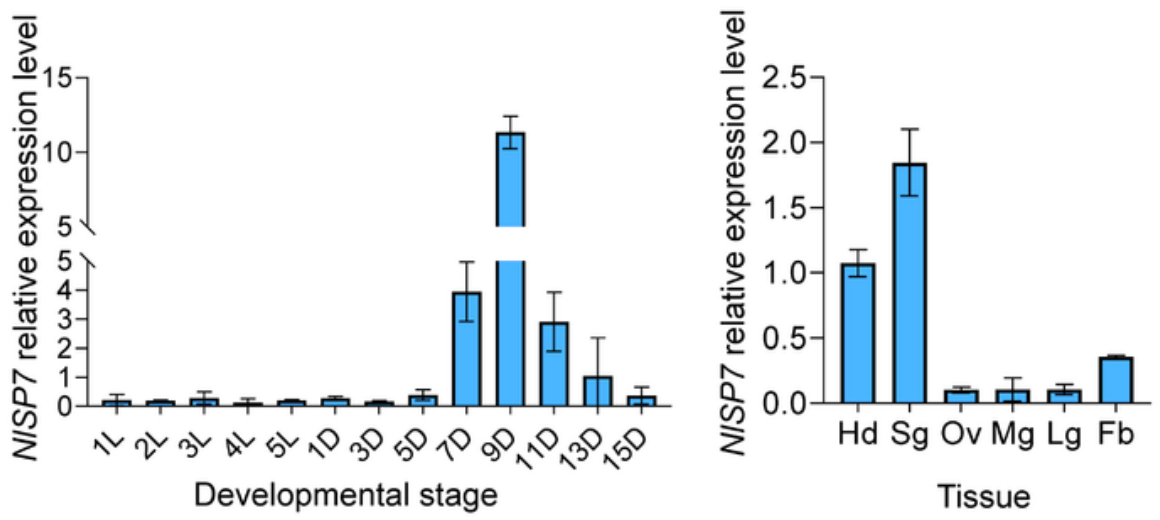

\section{Figure 1}

Characterization of NLSP7. A amino acid sequence of NLSP7. The solid underline indicates the signal peptide as predicted by SignalP 5.0. The asterisk $(*)$ indicates the stop codon. The different short-repeat regions are indicated by different colors. $B-C$ the bottom panels show the mean transcript levels $\pm S D(n=$ 30) of NLSP7 in whole bodies at various developmental stages (B) and in different tissues (C). RNA was extracted from nymphs at 1-5 days and female brachypterous brown planthoppers reared on Rathu 
Heenati rice at 1, 3, 5, 7, 9, 11, 13, and 15 days. Hd, head; Sg, salivary gland; Ov, ovary; Mg, midgut; Lg, leg; $\mathrm{Fb}$, fat body was extracted from BPH adults.

A
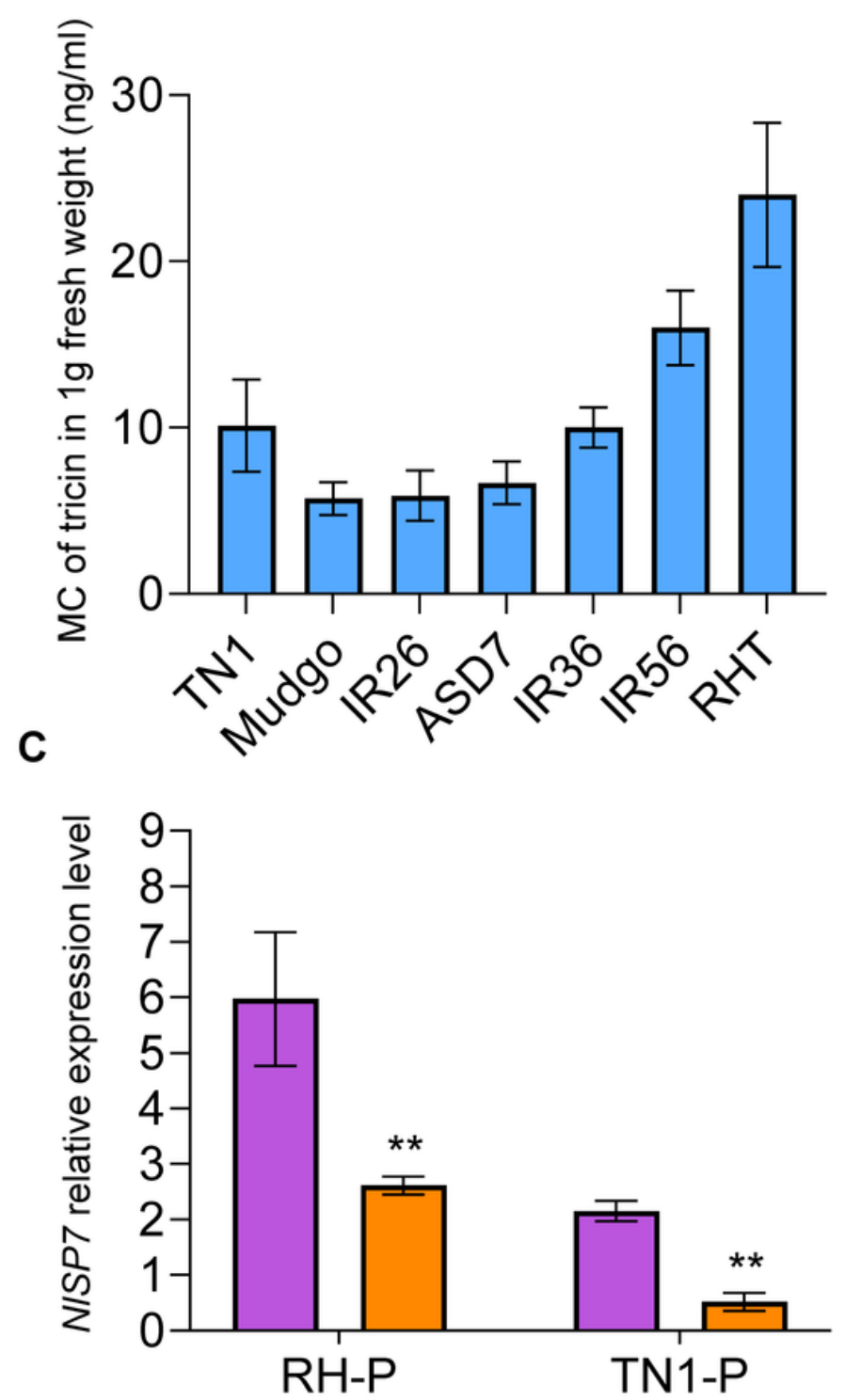

B
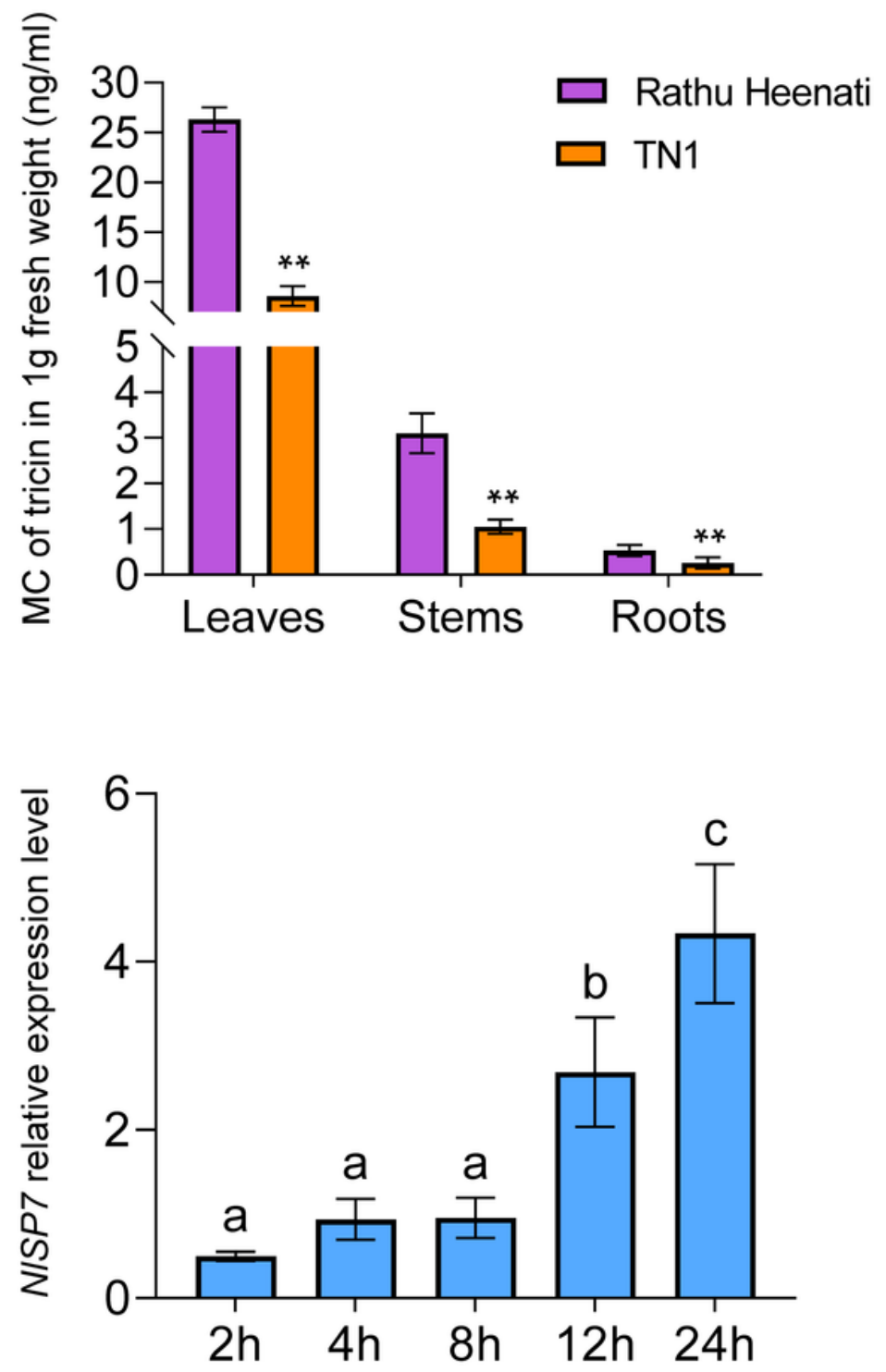

Figure 2

Relationship between NLSP7 and tricin. A tricin concentration $(\mathrm{ng} / \mathrm{g})$ analysis results at the three-leaf stage of seven rice varieties: Taichung Native 1 (TN1), Mudgo, IR26, ASD7, IR36, IR56, and Rathu Heenati $(\mathrm{RH})$ carrying different genes resistant to brown planthoppers. B expression level of tricin in the root, stem and leaf parts of RH and TN1 rice varieties. One g each of fresh root, stem, and leaf was ground, and the content of tricin in each part was measured using liquid chromatography-mass spectrometry. Data are represented as the mean \pm SD from three biological replicates. ${ }^{*}, \mathrm{P}<0.01$ (Student's t-test). C $\mathrm{q}-\mathrm{PCR}$ was used to determine the expression level of TN1-P and RH-P fed two different varieties of rice plants. D liquid diets sac-tricin was fed to brown planthoppers, then q-PCR was used to detect the expression level of NLSP7 at 2, 4, 8, 12, and 24 hours. The mean amounts of three biological replicates (mean \pm SD, $n=10$ ) 
are shown, with different letters indicating differences at five points $(2,4,8,12$, and $24 \mathrm{~h})(\mathrm{P}<0.01$, Duncan's multiple range test).

A

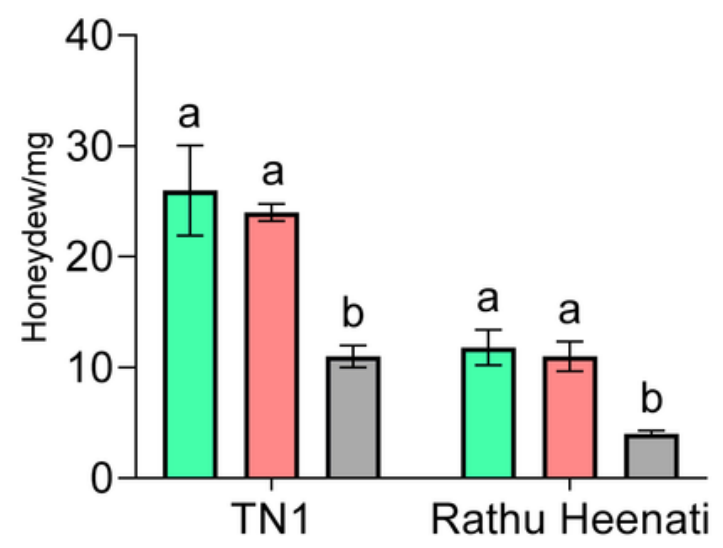

C

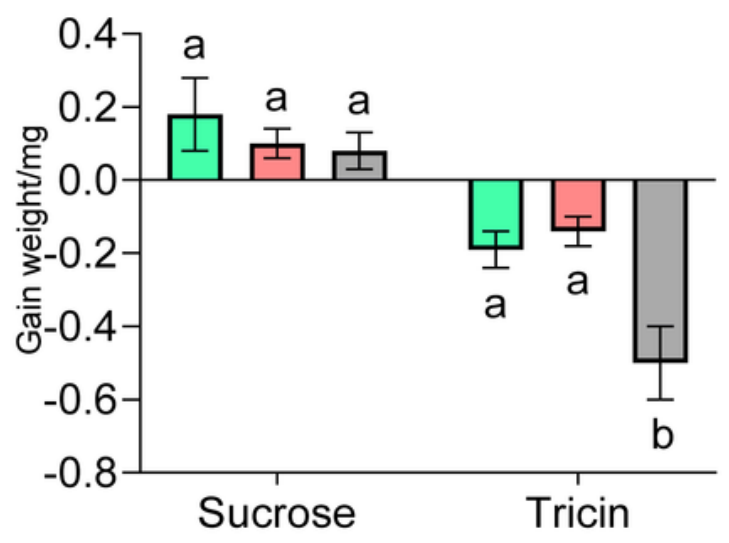

E

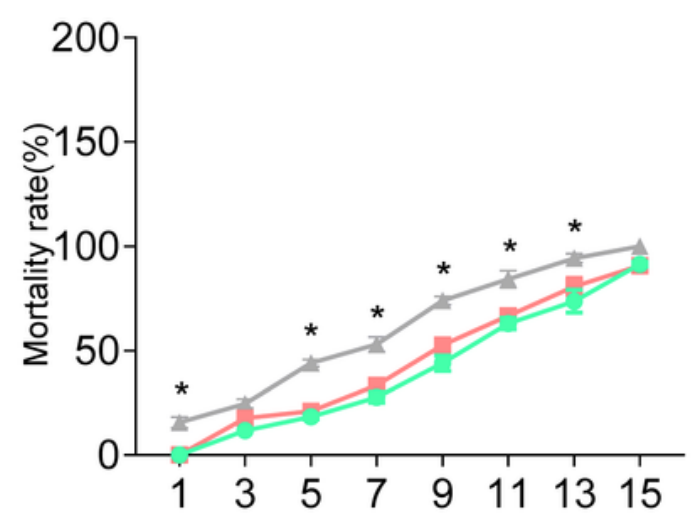

B

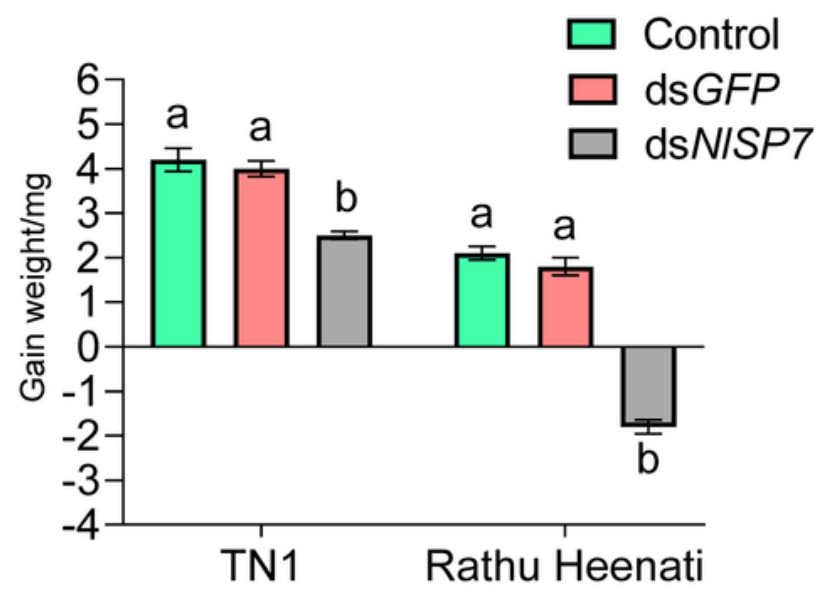

D

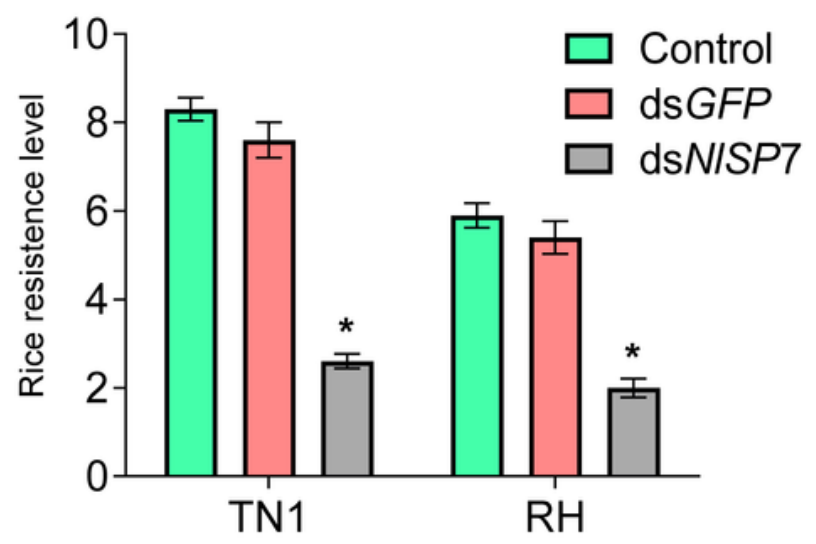

$\mathbf{F}$

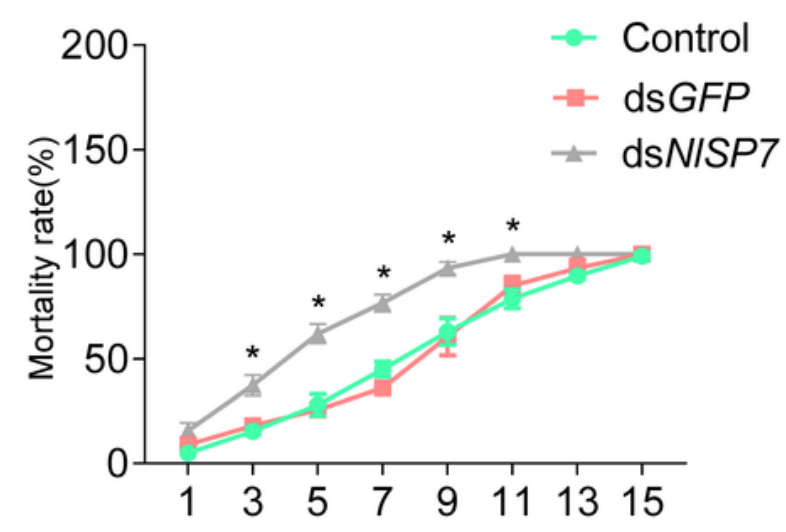

Figure 3

RNA interference (RNAi) effects on feeding of brown planthoppers (BPHs) exposed to three different treatments (control, injected with dsGFP, injected with dsNLSP7) and resistance levels of different rice varieties. A on Taichung Native 1 (TN1) and Rathu Heenati $(\mathrm{RH})$ rice varieties, the mean honeydew 
amounts excreted at $24 \mathrm{~h}$ by a female adult $\mathrm{BPH}$ that received three treatments. The experiment was repeated with 20 replicates. B weight gain of BPHs fed TN1 and $\mathrm{RH}$ rice varieties after injection with NLSP7. C mean weight of BPHs fed LDS-sucrose and LDS-tricin (mean $\pm S D, n=20$ ). Letters indicate significant differences $(P<0.01$, Duncan's multiple range test). $D$ rice resistance levels of BPHs fed TN1 and $\mathrm{RH}$ rice varieties. The experiment was repeated three times. Data are represented as the mean $\pm \mathrm{SD}$ $(\mathrm{P}<0.01$, Duncan's multiple range test). E mortality rate of $\mathrm{BPH}$ s fed $\mathrm{RH}$ rice, repeated 10 times, respectively. F mortality rate of BPHs fed an artificial diets with $100 \mathrm{mg} / \mathrm{I}$ tricin, repeated 10 times. Asterisks $\left(^{*}\right)$ indicate significant difference $(P<0.05$, Student's t-test).

A
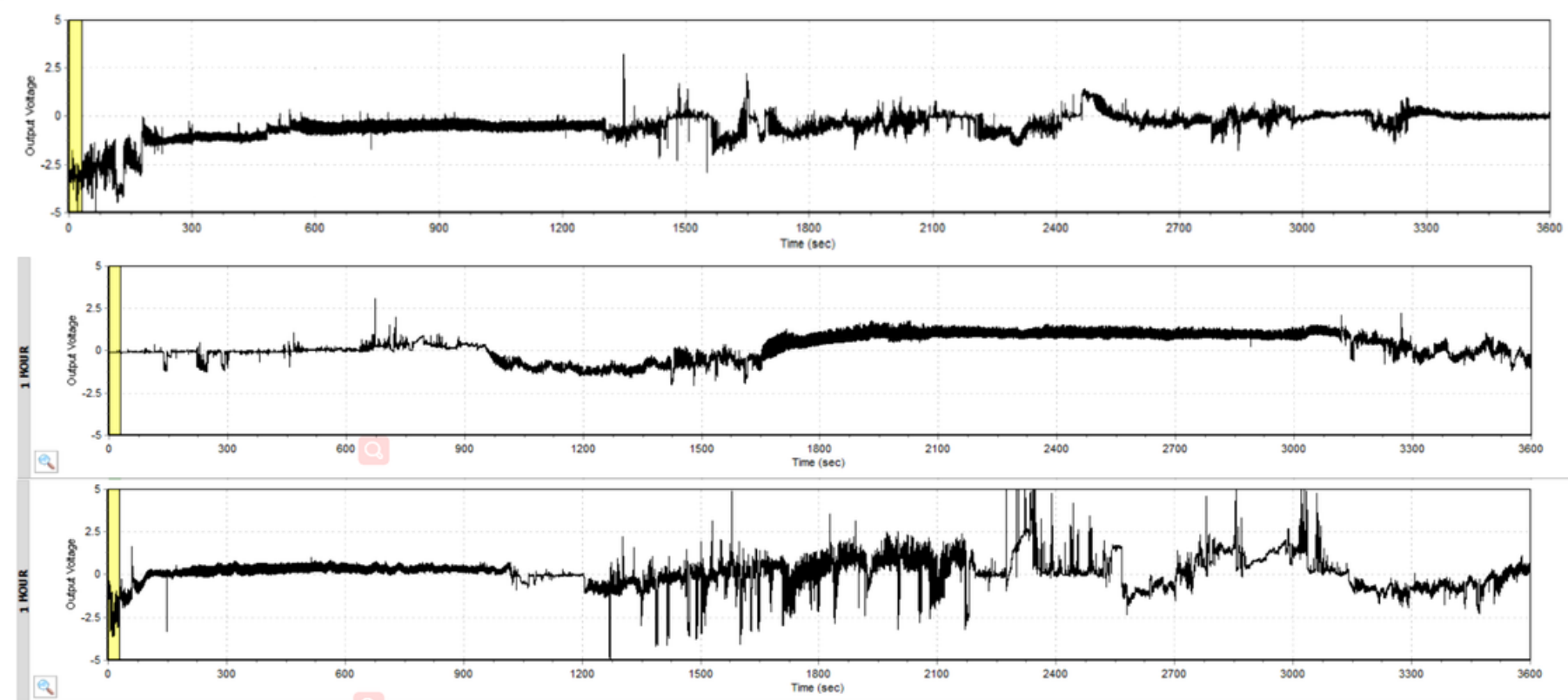

B

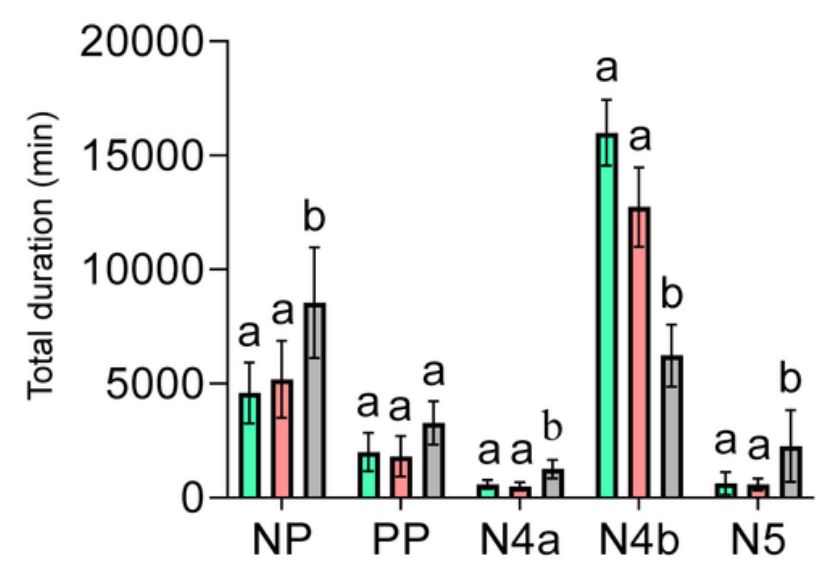

C

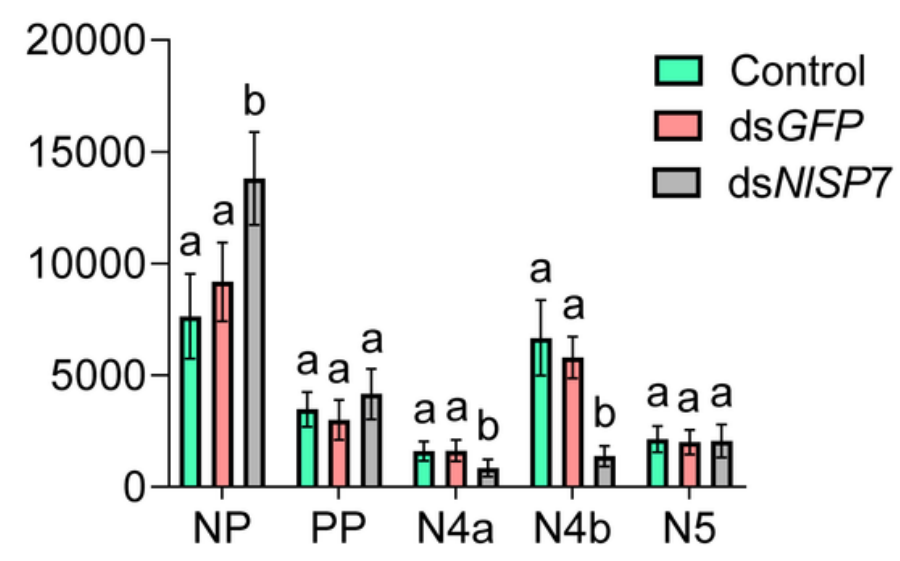

Figure 4

Reduced feeding on Taichung Native 1 (TN1) and Rathu Heenati (RH) rice varieties among three groups of brown planthoppers (control, injected with dsGFP, injected with dsNLSP7). A overall typical view of electrical penetration graph waveforms generated by feeding behavior of the three groups of brown planthoppers. B-C one-day-old brachypterous female adults in the three treatment groups. NP, 
nonpenetration; PP, pathway phase (N1 + N2 + N3), including penetration initiation (N1), salivation and stylet movement (N2), and extracellular activity near the phloem (N3); N4a, intracellular activity in the phloem region; N4b, phloem sap ingestion; N5, xylem phase. Duration of wavelength when each insect feeds on TN1 or RH rice. Electrical penetration graphs were recorded for $6 \mathrm{~h}$ per insect. Different letters indicate significant differences among the three treatment groups. Testing of each group was repeated 15 times $(P<0.01$, Duncan's multiple range test).

A
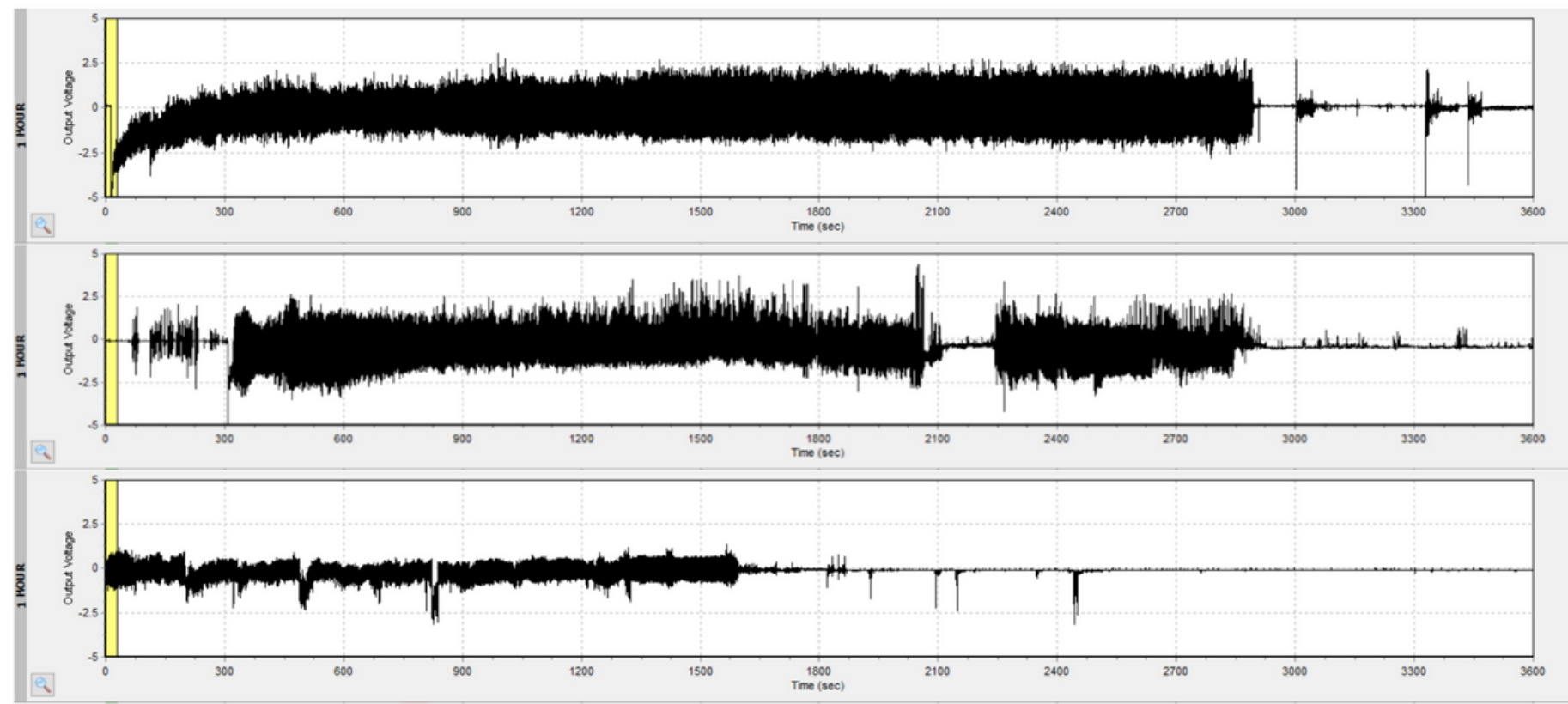

B

C
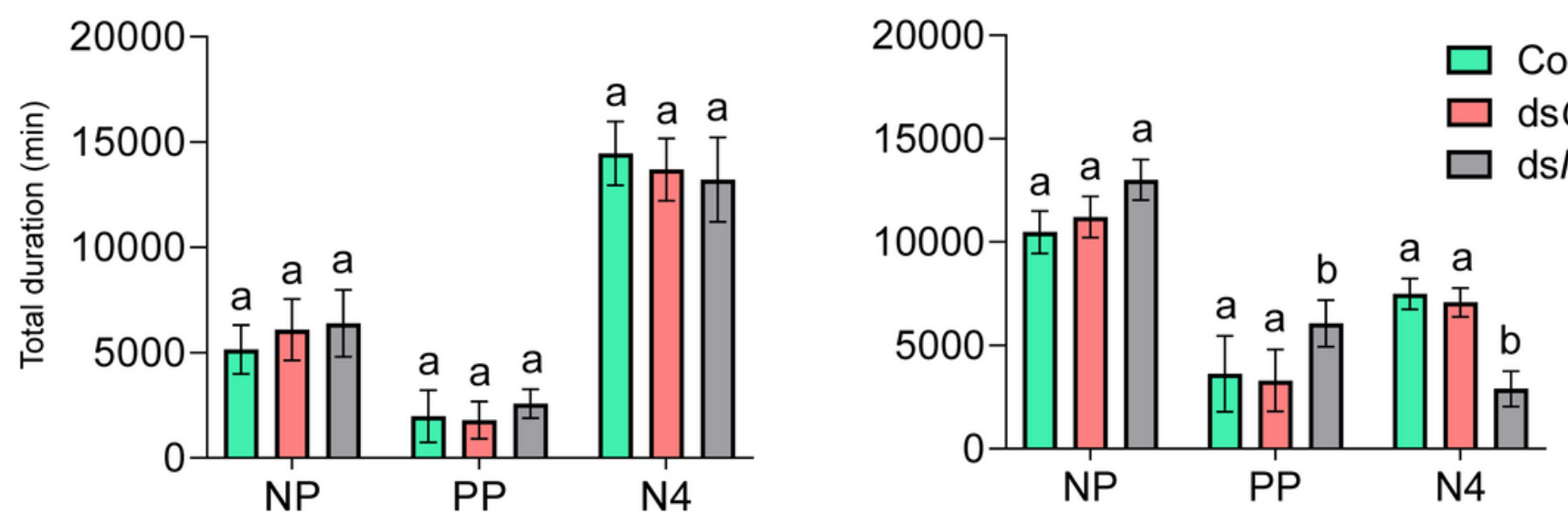

Figure 5

Brown planthoppers in three groups (control, injected with dsGFP, injected with dsNLSP7) exhibit reduced feeding on two artificial diets ( $0 \mathrm{mg} / \mathrm{l}$ tricin and $100 \mathrm{mg} / \mathrm{l}$ tricin). (A) Overall typical view of electrical penetration graph waveforms generated by the feeding behavior of brown planthoppers on artificial diets. B-C data collected from 1-day-old brachypterous female adults with different treatments. NP, nonpenetration; PP, pathway phase (N1 + N2 + N3), including penetration initiation (N1), salivation and stylet movement (N2), and extracellular activity near the phloem (N3); N4, sucrose or tricin solution 
ingestion. The duration of each wavelength when feeding on Liquid diets sac-tricin $(0 \mathrm{mg} / \mathrm{l})$ and Liquid diets sac-tricin $(100 \mathrm{mg} / \mathrm{l})$ is shown. Electrical penetration graph data were recorded for $6 \mathrm{~h}$ per insect. Different letters indicate significant differences among the three treatment groups. Testing of each group was repeated 15 times $(P<0.01$, Duncan's multiple range test).

A

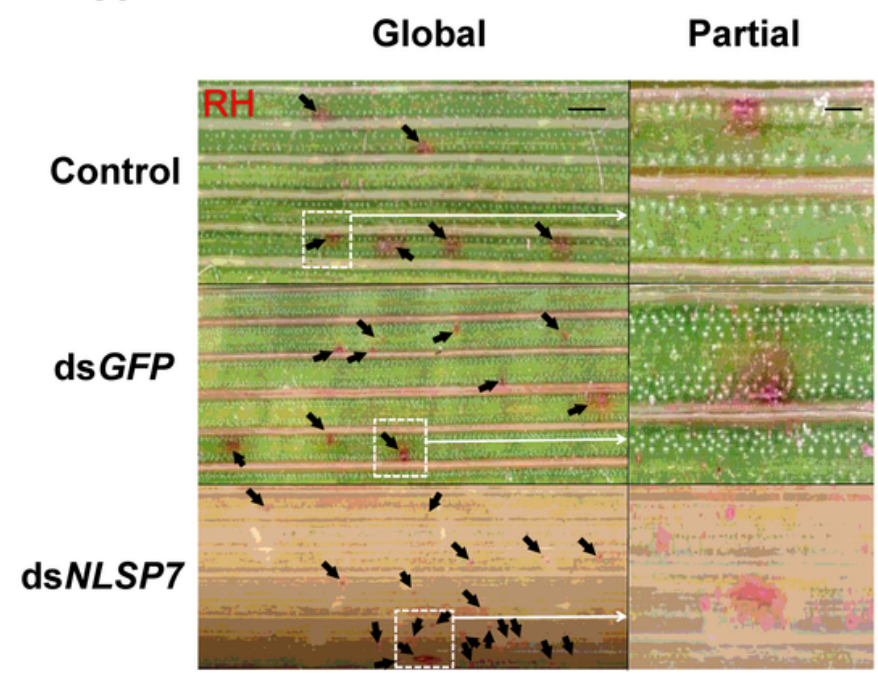

C

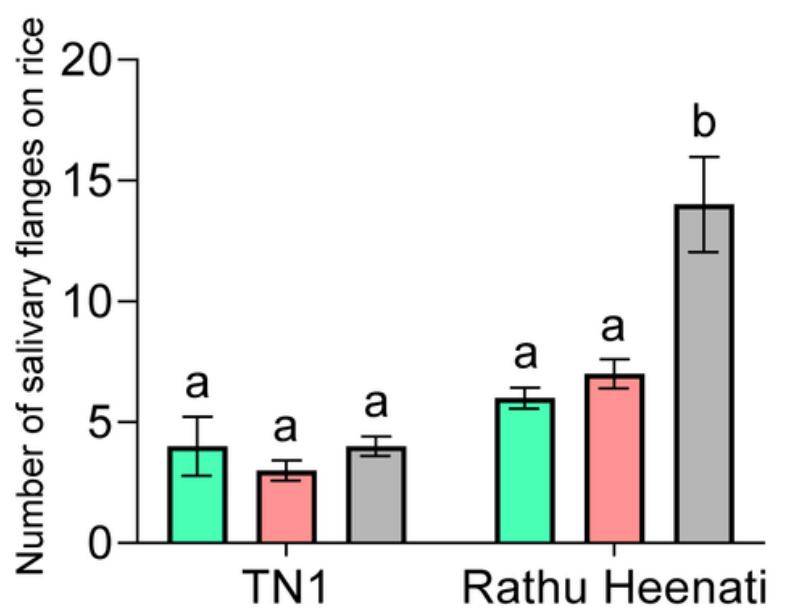

B

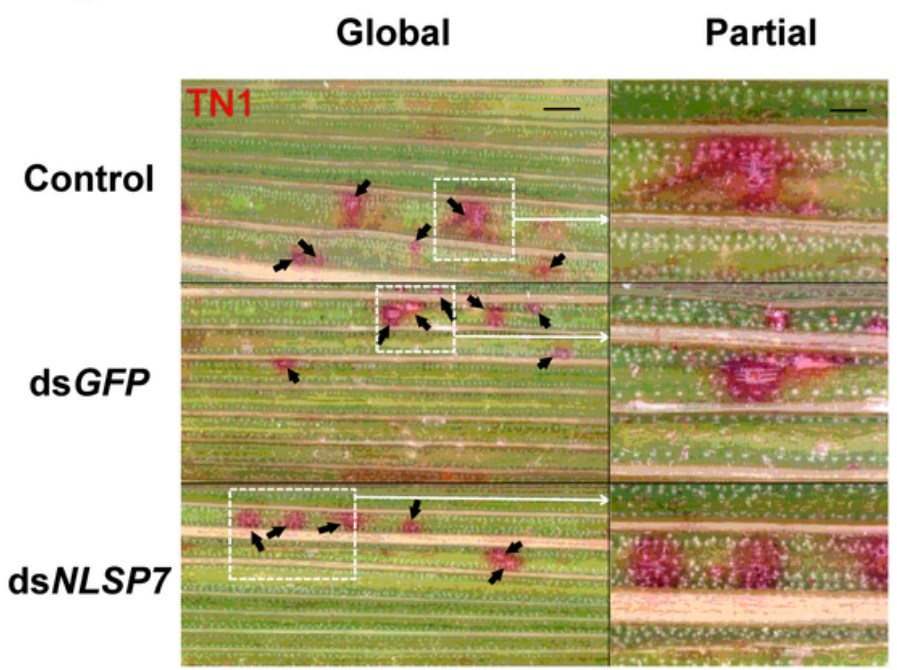

D

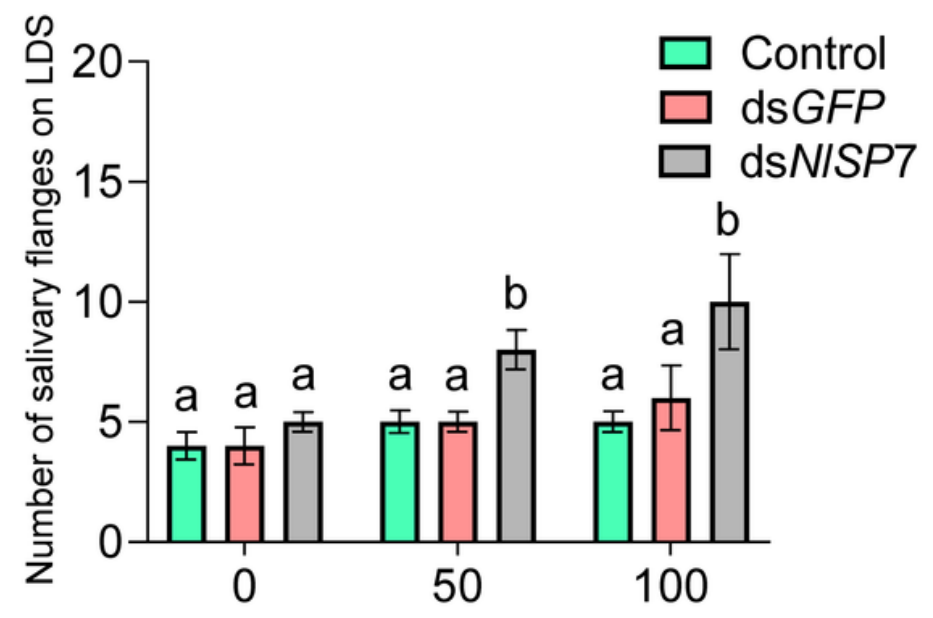

Figure 6

Number of salivary flanges created by brown planthoppers fed rice or an artificial diet, across different treatment groups (control, injected with dsGFP, injected with dsNLSP7). A-B images of salivary flanges produced on Rathu Heenati $(\mathrm{RH})$ and Taichung Native 1 (TN1) rice varieties. The global of graphs, bars = $200 \mu \mathrm{m}$. The partial of graphs, bars $=50 \mu \mathrm{m}$. C salivary flanges created by brown planthoppers fed different rice varieties. D salivary flanges from brown planthoppers fed artificial diets with different levels of tricin. Bars with different letters are significantly different among different tricin levels. Data are represented as means $\pm S D$, with 30 replications $(P<0.01$, Duncan's multiple range test). 
A

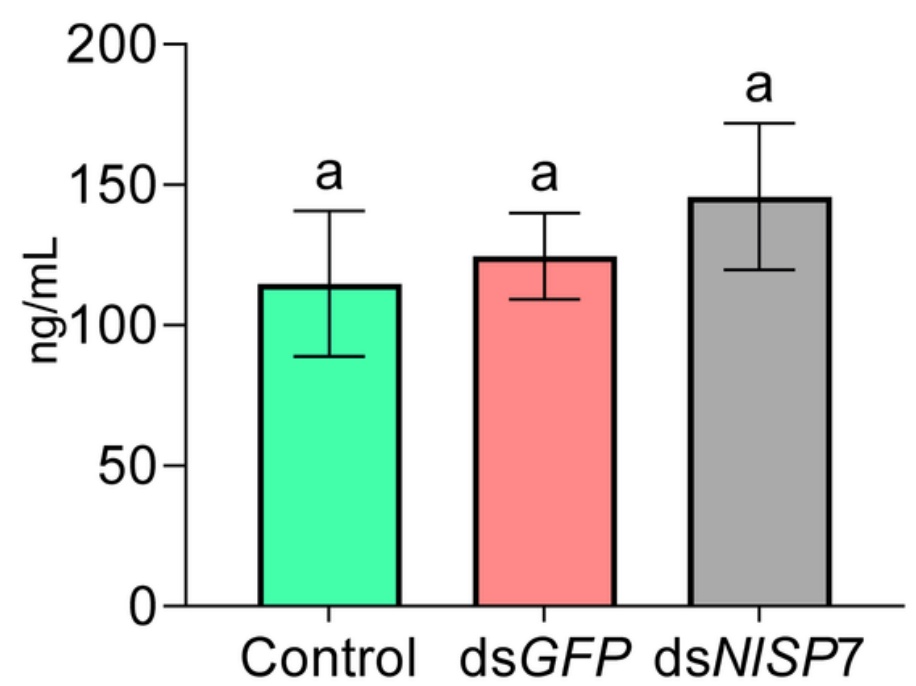

C

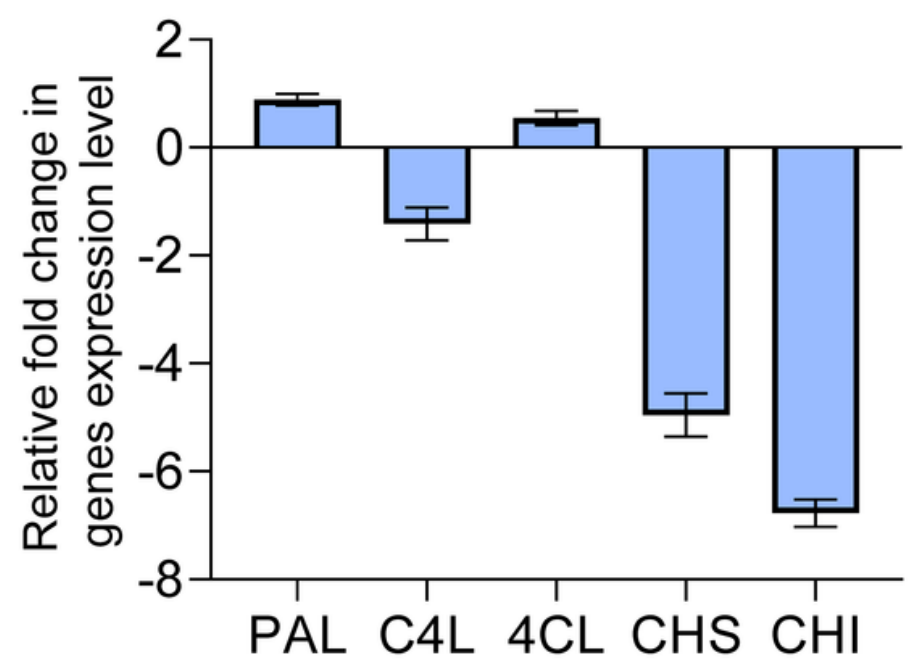

B

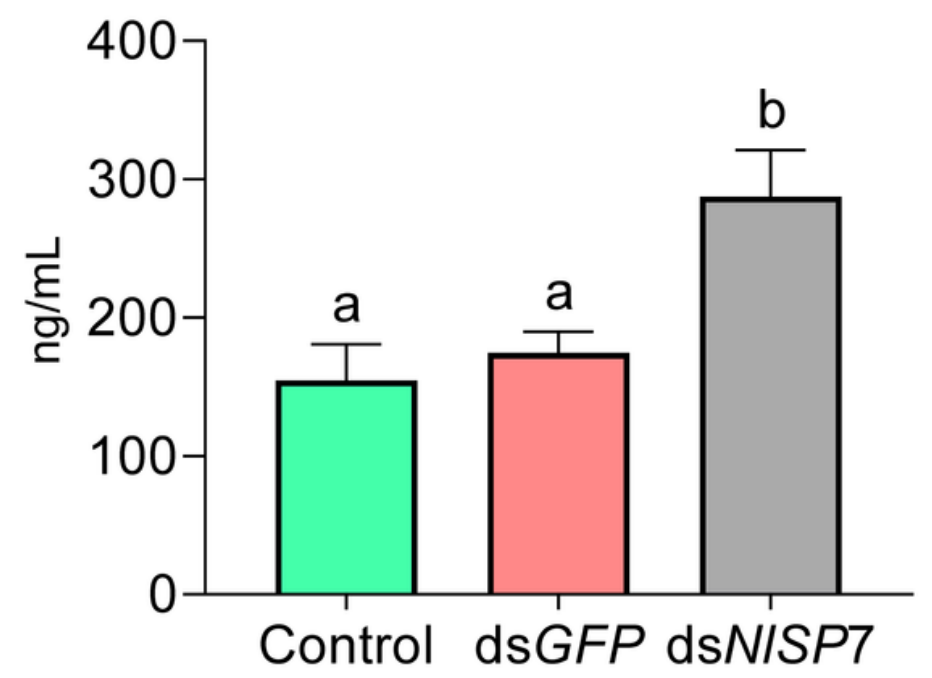

\section{Figure 7}

The effect of brown planthoppers in three groups (control, injected with dsGFP, injected with dsNLSP7) on the tricin content and tricin metabolic pathway genes of different rice varieties. A-B taichung Native 1 (TN1) and Rathu Heenati (RH) rice varieties fed on by brown planthoppers. After 3 days, the content of tricin was determined by liquid chromatography-mass spectrometry. Different letters represent significance. Data are represented as the mean $\pm S D$ from three biological replicates $(P<0.01$, Duncan's multiple range test). $\mathrm{C}$ the $\mathrm{q}-\mathrm{PCR}$ used to determine the expression level of tricin metabolic pathway genes in rice after being fed on by brown planthoppers. The experiment was repeated three times.

\section{Supplementary Files}


This is a list of supplementary files associated with this preprint. Click to download.

- Supportinginformation.docx 